\title{
Cognitive Test Score and 25-year Mortality Risk; Does Race Matter?
}

\section{Shervin Assari}

Department of Family Medicine, Charles R. Drew University of Medicine and Science, Los Angeles, California 90095, USA

\section{Abstract}

Objectives: Despite our knowledge of the effect of cognitive test scores on subsequent risk of mortality, few studies have compared Blacks and Whites for this association. The current study was conducted on Black-White differences in the magnitude of the association between baseline cognitive test score and all-cause mortality in a nationally representative sample of adults in the United States over 25 years. Methods: We used data from the Americans' Changing Lives Study, 1986-2011, a national prospective cohort in the U.S. The study followed 3361 adults (2205 White and 1156 Blacks), age 25 and older, for up to 25 years. The independent variable was cognitive test score measured at baseline (1986) using the 4-item version of the Short Portable Mental Status Questionnaire, treated in two different ways (as a dichotomous and as a continuous variable). The dependent variable was time to death (due to all causes) during the follow-up period. Covariates included baseline age, gender, education, income, number of chronic diseases, self-rated health, and depressive symptoms. Race (Black vs. White) was the focal effect modifier. We used a series of Cox proportional hazards models in the total sample, and by race, in the absence and presence of health variables. Results: Overall, cognitive test score predicted mortality risk. A significant interaction was found between race and baseline cognitive test score, suggesting that baseline cognitive test score has a weaker protective effect against all-cause mortality for Blacks un comparison to Whites. In race-stratified models, cognitive test scores at baseline predicted risk of all-cause mortality for Whites but not Blacks, in the absence and presence of baseline socio-economic and health variables. The results were similar regardless of how we treated baseline cognitive test scores. Conclusions: In the United States, baseline cognitive test score has a weaker protective effect against all-cause mortality over a long period of time for Blacks than Whites. The finding is in line with the Minorities Diminished Returns theory and is probably due to structural and interpersonal racism.

\section{Keywords: African Americans, Blacks, Cognition, Cognitive test score, Death, Ethnic groups, Ethnicity, Life expectancy, Mortality, Population groups, Race, Whites}

\section{Introduction}

It is well established that particular cognitive abilities decline with age, ${ }^{[1]}$ and this decline is associated with morbidity ${ }^{[2]}$ and mortality. ${ }^{[3]}$ Longitudinal studies have shown that cognitive test scores at baseline predicts future morbidity ${ }^{[4]}$ and mortality. ${ }^{[5]}$ Due to the association between baseline cognitive test score and life expectancy, ${ }^{[3]}$ high cognitive function is expected to protect populations against the risk for premature mortality. ${ }^{[6]}$

Despite the well-defined link between baseline cognitive function and subsequent all-cause mortality, ${ }^{[3,7-9]}$ it is not known whether or not diverse population groups differ in this relationship, as very few studies have ever tested predictive ability of cognitive limitation on long-term mortality among racial and ethnic minority populations. Of the few studies that have been conducted among racial and ethnic minorities, most have enrolled Hispanics. ${ }^{[6,10]}$ In a study among older Mexican Americans, baseline and also 2-year decline in cognitive functioning independently predicted risk of all-cause mortality. ${ }^{[6]}$ In another study among Mexican Americans, although frailty and cognitive test score were risk factors for mortality separately, the effect

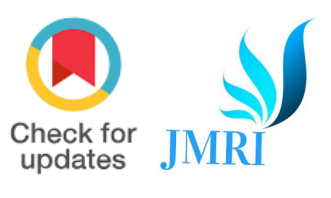

How to cite this article: Assari S. Cognitive test score and 25-year mortality risk; Does race matter?. J Med Res Innov. 2020;4(2):e000213.

DOI: 10.32892/jmri.213

Publication history: Received: 02-02-2020 Accepted: 23-04-2020 Published: 23-04-2020

Editor: Dr. Varshil Mehta

Copyright: Assari S, This is an open-access article distributed under the terms of the Creative Commons Attribution License CC-BY 4.0., which permits unrestricted use, distribution, and reproduction in any medium, provided the original author and sources are credited.

Funding: Assari is funded by the NIH awards U54CA229974, U54MD008149, U54MD008149, R25MD007610, U54MD007598, U54TR001627, and CA201415-02. The Americans' Changing Lives (ACL) study was supported by the NIH grant AG018418. $\mathrm{NIH}$ is not responsible for the data collection or analyses represented in this article. The ACL study was conducted by the Institute of Social Research, University of Michigan.

Conflict of Interest: NIL

MK Medkrux 
of cognitive impairment on mortality disappeared by controlling for frailty. ${ }^{[10]}$ Unfortunately, compared to Whites or even Hispanics, less is known about this association among Black Americans. ${ }^{[1]}$

\section{Aim}

To fill the gap in the literature, and to better understand whether race interferes with the role of cognitive test score on life expectancy in the United States, we conducted this study on Black-White differences in the magnitude of the protective effect of baseline cognitive test score on subsequent all-cause mortality, using a nationally representative sample. In line with the Minorities Diminished returns theory ${ }^{[85-93]}$ and also some other empirical evidence on ethnic differences in predictive role of baseline risk and protective factors on long-term outcomes, ${ }^{[12-14]}$ we hypothesized a stronger effect for Whites compared to Blacks.

\section{Methods \\ Design and setting}

The Americans' Changing Lives ( $A C L$ ) study ( $A C L$; 1986-2011) is a 25-year prospective longitudinal study of American adults (aged 25 years and over). This study recruited a national (representative) sample of U.S. residents. Although more information on their methods and sampling procedure is published elsewhere, we briefly explain some of the aspects of the study here. ${ }^{[15-17]}$

\section{Ethics}

The University of Michigan (UM) Ethical committee (also known as the Institutional Review Board; UMIRB) approved the study protocol. Written informed consent was received from all participants of the ACL.

\section{Participants and sampling}

Using stratified multistage sampling, the ACL recruited a probability sample of U.S. adults who were at least 25 years old. The study participants were 3617 adults who were living in the continental U.S. The only other inclusion criteria for the ACL was being noninstitutionalized. The ACL oversampled older adults (age 60 years or more) and African Americans/Blacks. Wave 1 data included $70 \%$ and $68 \%$ of the sampled households and individuals respectively.

\section{Analytical sample}

The current analysis is only limited to Europeans/ Whites and African Americans/Blacks (analytic $n=3361$ who were either White $[n=2205]$ or Black $[n=1,156])$.

\section{Process}

All the baseline data were gathered through faceto-face interviews (first wave; year 1986). Death certificates were used to assess the date of death.

\section{Measures}

Baseline demographic factors, socio-economic characteristics, self-rated health (SRH), depressive symptoms, and chronic medical conditions were measured in 1986 to adjust for possible confounding. The main outcome was time to death (due to any causes) during a 25-year follow-up period.

\section{Race/Ethnicity}

In this study, race was the focal effect modifier, treated as a dichotomous levels: Black 1 versus White 0 (referent category). Race and ethnicity were self-identified using multiple items in wave 1 of data collection (1986). Responses of these items informed our racial/ethnic categories: "NonHispanic White," "Non-Hispanic Black," "NonHispanic Native American," "Non-Hispanic Asian," and "Hispanic." The current analysis only included two racial/ethnic groups: Non-Hispanic White and Non-Hispanic Black. Thus, this analysis does not include any Hispanic individuals.

\section{Cognitive test score}

The ACL survey adopted the 4-item Short Portable Mental Status Questionnaire (SPMSQ) for the measurement of cognitive test scores. The SPMSQ assesses the following aspects of cognition: knowledge of current events, memory, as well as the ability to perform some basic mathematical tasks. The SPMSQ is designed to measure individuals' cognitive performance from community and institutionalized samples. ${ }^{[18,19]}$ The SPMSQ measure had the following four items: (a) "What is the date today - month, day, and year?" (b) "What day of the week is it?" (c) "What is the name of the president of the United States?" (d) "Subtract 3 from 20 and tell me the number you get. Then, keep subtracting 
3 from this number and each new number you get, telling me the results as you go (Stop when the answer is 2 or less)." Each item was coded as 0 "correct" or 1 "incorrect," with a total cognitive score ranging between 0 and 4 , with a higher score indicating worse cognitive performance. ${ }^{[20]}$ A score of 2 or more (participants who had $2+$ errors) was considered a low cognitive score. ${ }^{[21,22]}$

\section{Demographics}

Demographic characteristics included age (a continuous variable) and gender (a dichotomous variable with male as the referent category) collected in 1986.

\section{Socioeconomic status (SES)}

This study collected baseline educational attainment (years of schooling) and income level (an ordinal variable) collected in 1986 as the SES measures.

\section{Number of chronic diseases}

The baseline number of chronic medical conditions was measured based on self-reported data. Individuals were asked if a health-care provider had ever informed that they had any of the following seven chronic conditions: Diabetes, hypertension, chronic lung disease, stroke, heart disease, cancer, and arthritis. Responses for whether the individual had a chronic conditions was on a dichotomous scale (yes/no) for each condition. The number of chronic diseases was an interval variable ranging from 0 to $7 .{ }^{[16,17]}$

\section{SRH}

Participants' SRH was measured using a single item with the following five categories: Excellent, very good, good, fair, and poor. In this study, SRH was treated as a dichotomous measure. The five categories were collapsed into the following two categories: Excellent/very good/good 0 versus fair/poor 1. This cutoff is compliant with the literature. ${ }^{[23,24]}$ The single item SRH measure has shown very high test-retest reliability as well as validity. Poor SRH is a strong predictor of mortality risk net of confounders. ${ }^{[25,26]}$

\section{Depressive symptoms}

Symptoms of depression were captured using the 11-item Center for Epidemiological StudiesDepression scale (CES-D). ${ }^{[27]}$ CES-D measures the degree to which the participant felt depressed, sad, lonely, restless, etc. Some of the CES-D items are positively worded, which are reverse-coded. Short versions of the CES-D scale have acceptable reliability and validity. ${ }^{[28-30]}$ Items are on a response scale ranging from 1 to 3 . As a result, the CES-D score in this study ranged from 11 to 33, with a higher score indicated more depressive symptoms.

\section{Mortality}

Mortality data were primarily gathered either from death certificates or from the National Death Index (NDI). Overall, 1402 deceased participants were detected, while 1959 individuals survived. Collected death data included the date of death, as well as primary and underlying causes of death. In the United States, death certificates are filled out by doctors as soon as possible after individuals are deceased. The cause of death was not used for this study. For the 1402 deceased cases, the month of death was obtained from the NDI or death

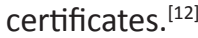

\section{Statistical analysis}

Due to the survey design, we used Stata-13 (Stata Corp., College Station, Texas, United States) to calculate design-based standard errors (SE) based on sampling and non-response weights at baseline. Taylor series linearization was used to estimate SEs. Survey linear, logistic, and Cox proportional hazards models were used for data analysis. Hazard ratios $(H R)$, Standardized regression coefficient (B), and odds ratios (OR) with their SEs and 95\% confidence intervals are reported.

In the first step, and to test the cross-sectional association between baseline age, gender, education, income, number of chronic diseases, $\mathrm{SRH}$, and symptoms of depression with baseline cognitive test score, we used three logistic regression models (Model 1-3) where the outcomes was cognitive test score treated as a dichotomous measure. For the same aim, we also ran three linear regressions (Model 4-6), where the outcome was cognitive test score treated as a continuous measure. First, we used the pooled sample with the main effect of race in the model (Model 1-4). Then, we ran models that were stratified by race/ethnicity to test if demographic, socio-economic, and health factors predict cognitive test scores (a continuous score) at baseline among Whites (Model 2-5) and Blacks (Model 3-6).

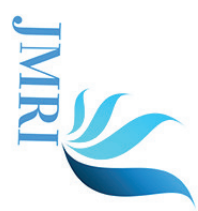


In the next step, and to assess the effect of cognitive test scores on mortality, we used a series of Cox proportional hazards models. Baseline cognitive test score, the independent variable, was measured in 1986, and was treated in two ways: A dichotomous and a continuous measure. The dependent variable was time to death for any cause between 1986 and 2011. Covariates included age, gender, education, income, number of chronic diseases, $\mathrm{SRH}$, and symptoms of depression, which were entered into the model in a step-wise fashion. First, we used the pooled sample of Blacks and White to evaluate the effects of baseline cognitive test scores on mortality, while covariates were being added gradually. Then, we ran models stratified by race to test if a cognitive test score at baseline differently predicts time to death over up to 25 years of follow-up among Whites and Blacks.

\section{Results}

Table 1 presents a summary of descriptive statistics for all study variables overall and based on race. Age and gender were not significantly different between racial groups. Blacks had significantly lower education and income and worse $\mathrm{SRH}$, and displayed greater depressive symptoms and more chronic medical conditions. Blacks also had higher cognitive test scores at baseline than Whites.

Table 2 shows three logistic and three linear regressions with binary and continuous cognitive test scores as the outcomes. With a similar pattern among Whites and Blacks, age, education, income, and depressive symptoms, but not gender and number of chronic medical conditions, was associated with baseline cognitive impairment. Whites and Blacks, however, showed a different patterns of associations between SRH and depressive symptoms with cognitive test scores at baseline.

Table 3 summarizes the results of several proportional hazards models that were performed in the pooled sample (Models 1-3, 8-10), as well as Whites (Model $4,5,11,12$ ) and Blacks (Model 6, 7, 13, 14), in the absence of health conditions in the model. According to this table, in the pooled sample, higher baseline cognitive test score was correlated with higher mortality risk. There was also a significant interaction between race and baseline cognitive test scores on the outcome, suggesting that the effect of baseline cognitive test score on mortality is stronger for

Table 1: Descriptive statistics for the analytic sample, stratified by race, and overall

\begin{tabular}{|c|c|c|c|c|c|c|}
\hline \multirow[t]{2}{*}{ Characteristics } & All & \multirow[t]{2}{*}{$95 \% \mathrm{Cl}$} & Whites & \multirow[t]{2}{*}{$95 \% \mathrm{Cl}$} & Blacks & \multirow[t]{2}{*}{$95 \% \mathrm{Cl}$} \\
\hline & Mean (SE) & & Mean (SE) & & Mean (SE) & \\
\hline Age & $47.77(0.534)$ & $46.69-48.84$ & $47.96(0.601)$ & $46.75-49.17$ & $46.33(0.717)$ & $44.89-47.78$ \\
\hline Education* & $12.53(0.096)$ & $12.34-12.73$ & $12.69(0.105)$ & $12.48-12.90$ & $11.37(0.233)$ & $1.90-11.84$ \\
\hline Income* & $5.41(0.093)$ & $5.22-5.60$ & $5.57(0.101)$ & $5.36-5.77$ & $4.25(0.183)$ & $3.88-4.62$ \\
\hline CMC* & $0.79(0.028)$ & $0.74-0.85$ & $0.78(0.031)$ & $0.71-0.84$ & $0.91(0.052)$ & $0.81-1.02$ \\
\hline \multicolumn{7}{|l|}{$\mathrm{SRH}^{*}$} \\
\hline Good-Excellent & $85.06(0.008)$ & $83.33-86.64$ & $85.97(0.009)$ & $84.15-87.60$ & $78.38(0.017)$ & $74.68-81.68$ \\
\hline Poor-Fair & $14.94(0.008)$ & $13.36-16.67$ & $14.03(0.009)$ & $12.40-15.85$ & $21.62(0.017)$ & $18.32-25.32$ \\
\hline CES-D* & $-0.03(0.025)$ & $-0.08-0.02$ & $-0.07(0.025)$ & $-0.13--0.02$ & $0.28(0.051)$ & $0.18-0.38$ \\
\hline \multirow[t]{2}{*}{ Cognitive test score $*$} & $0.70(0.03)$ & $0.63-0.76$ & $0.65(0.04)$ & $0.58-0.73$ & $1.01(0.05)$ & $0.91-1.11$ \\
\hline & $\%$ (SE) & $95 \% \mathrm{Cl}$ & $\%$ (SE) & $95 \% \mathrm{Cl}$ & $\%$ (SE) & $95 \% \mathrm{Cl}$ \\
\hline \multicolumn{7}{|l|}{ Gender } \\
\hline Male & $47.26(0.012)$ & $44.86-49.68$ & $47.82(0.013)$ & $45.12-5.52$ & $43.18(0.022)$ & $38.79-47.69$ \\
\hline Female & $52.74(0.012)$ & $5.32-55.14$ & $52.18(0.013)$ & $49.48-54.88$ & $56.82(0.022)$ & $52.31-61.21$ \\
\hline \multicolumn{7}{|l|}{ Cognitive test score* } \\
\hline Intact & $84.37(0.01)$ & $82.29-86.25$ & $86.14(0.01)$ & $83.77-88.21$ & $71.38(0.02)$ & $67.53-74.95$ \\
\hline Impaired & $15.63(0.01)$ & $13.75-17.71$ & $13.86(0.01)$ & $11.79-16.23$ & $28.62(0.02)$ & $25.05-32.47$ \\
\hline
\end{tabular}

${ }^{*} P<0.05$ for all comparisons between Blacks and Whites; CMC: Chronic Medical Conditions; CES-D: Center for Epidemiologic Studies Depression. Cl: Confidence interval, SRH: Self-rated health, SE: Standard error 
Assari: Race, cognition, and mortality

Table 2: Predictors of baseline cognition impairment based on race using logistic and linear regression

\begin{tabular}{|c|c|c|c|c|c|c|}
\hline Characteristics & All & & Whites & & Blacks & \\
\hline & 95\% Cl OR (SE) & & 95\% Cl OR (SE) & & 95\% Cl OR (SE) & \\
\hline & Model 1 & & Model 2 & & Model 3 & \\
\hline $\begin{array}{l}\text { Cognitive } \\
\text { function as a } \\
\text { continuous } \\
\text { measure }\end{array}$ & & & & & & \\
\hline Race (Black) & $2.01(0.27)^{* * *}$ & $1.52-2.64$ & - & - & - & - \\
\hline Gender (Female) & $0.87(0.10)$ & $0.69-1.10$ & $0.81(0.12)$ & $0.60-1.10$ & $1.16(0.22)$ & $0.79-1.71$ \\
\hline Age & $1.01(0.00)^{* * *}$ & $1.01-1.02$ & $1.01(0.00)^{* * *}$ & $1.01-1.02$ & $1.02(0.01)^{*}$ & $1.00-1.03$ \\
\hline Education & $0.49(0.09)^{* * *}$ & $0.34-0.70$ & $0.46(0.10)^{* * *}$ & $0.30-0.70$ & 0.63 (0.15)\# & $0.38-1.03$ \\
\hline Income & $0.92(0.02)^{* *}$ & $0.88-0.97$ & $0.92(0.03)^{* *}$ & $0.87-0.98$ & $0.93(0.04)$ & $0.86-1.02$ \\
\hline CMC & $1.01(0.07)$ & $0.88-1.15$ & $1.00(0.08)$ & $0.85-1.16$ & $1.08(0.11)$ & $0.87-1.33$ \\
\hline SRH (Poor/Fair) & $1.18(0.18)$ & $0.86-1.61$ & $1.10(0.22)$ & $0.74-1.64$ & 1.47 (0.30)\# & $0.98-2.21$ \\
\hline CESD-11 & $1.14(0.07)^{*}$ & $1.99-1.31$ & $1.14(.08)^{\#}$ & $0.99-1.31$ & $1.15(0.12)$ & $0.93-1.42$ \\
\hline Cognitive & B (SE) & $95 \% \mathrm{Cl}$ & B (SE) & $95 \% \mathrm{Cl}$ & B (SE) & $95 \% \mathrm{Cl}$ \\
\hline $\begin{array}{l}\text { function as a } \\
\text { dichotomous } \\
\text { measure }\end{array}$ & Model 4 & & Model 5 & & Model 6 & \\
\hline Race (Black) & $0.24(0.06)^{* * *}$ & $0.12-0.36$ & - & - & - & - \\
\hline Gender (Female) & $-0.01(0.04)$ & $-0.09-0.07$ & $-0.03(0.05)$ & $-0.12-0.06$ & $0.16(0.07)^{*}$ & $0.01-0.30$ \\
\hline Age & $0.01(0.00)^{* * *}$ & $0.00-0.01$ & $0.01(0.00)^{* *}$ & $0.00-0.01$ & $0.01(0.00)^{* *}$ & $0.00-0.02$ \\
\hline Education & $-0.38(0.07)^{* * *}$ & $-0.52--0.24$ & $-0.38(0.08)^{* * *}$ & $-0.54--0.22$ & $-0.34(0.10)^{* *}$ & $\begin{array}{c}-0.54- \\
-0.13\end{array}$ \\
\hline Income & $-0.02(0.01)^{* * *}$ & $-0.04--0.01$ & $-0.02(0.01)^{* *}$ & $-0.04--0.01$ & $-0.04(0.02)^{*}$ & $-0.07-0.00$ \\
\hline $\mathrm{CMC}$ & $0.00(0.02)$ & $-0.05-0.04$ & $-0.01(0.02)$ & $-0.05--0.04$ & $0.01(0.05)$ & $-0.09-0.10$ \\
\hline SRH (Poor/Fair) & $0.09(0.05)$ & $-0.01-0.19$ & $0.05(0.06)$ & $-0.07-0.16$ & $0.30(0.08)^{* * *}$ & $0.14-0.46$ \\
\hline CESD-11 & $0.06(0.02)^{* *}$ & $0.02-0.09$ & $0.05(0.02)^{* *}$ & $0.01-0.09$ & $0.09(0.04)^{*}$ & $0.01-0.17$ \\
\hline
\end{tabular}

$\# P<0.1,{ }^{*} P<0.05,{ }^{*} P<0.01,{ }^{*} * P<0.001, \mathrm{CMC}$ : Chronic Medical Conditions; CES-D: Center for Epidemiologic Studies Depression.

$\mathrm{Cl}$ : Confidence interval, SRH: Self-rated health, SE: Standard error, OR: Odds ratios

Whites than Blacks, when demographic and baseline socio-economic factors are controlled (Model 4, 5, 11, 12). Race-specific models showed that baseline cognitive test score only predicts mortality among Whites but not Blacks.

Table 4 provides the results of Cox proportional hazards models in the pooled sample (Models 1,2,5,6) and among Whites (Models 3, 7) and Blacks (Models $4,8)$, while health variables are also controlled. In this table, in the overall (pooled) sample, a statistically significant interaction effect was found between race and baseline cognitive test scores suggesting a stronger effect of baseline cognitive test score on mortality for Whites relative to Blacks (Models 2,
6). Race-specific models confirmed our finding, as cognitive test scores at baseline predicted mortality among Whites but not Blacks.

\section{Discussion}

Our study had three main findings. On the main aim of the study, major Black-White differences were found in the predictive role of baseline cognitive function on long-term mortality. The study also showed lower cognitive function at baseline for Blacks, and Black and White similarities in role of age and socio-economic status on cognitive function. These findings contribute to the existing literature on health disparities in the United States, 


\begin{tabular}{|c|c|c|c|c|c|c|c|c|c|c|c|c|c|c|}
\hline ถั & & 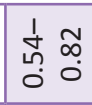 & i & 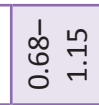 & $\begin{array}{ll}1 & 1 \\
0 & 0 \\
0 & 8 \\
0 & 0\end{array}$ & $\begin{array}{ll}\frac{1}{d} \\
\substack{\infty \\
0 \\
0}\end{array}$ & ' & & $\begin{array}{ll}1 & 0 \\
1 & 0 \\
0 & 0 \\
0 & 0 \\
0\end{array}$ & \begin{tabular}{ll}
1 & $\infty$ \\
0 & $\infty$ \\
\hdashline & $\stackrel{-}{-}$
\end{tabular} & 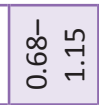 & $\begin{array}{ll}1 & 0 \\
0 & 0 \\
0 & 0 \\
0 & 0 \\
0\end{array}$ & 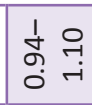 & ' \\
\hline 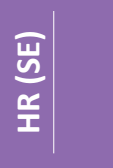 & $\begin{array}{l}\text { No } \\
\frac{\pi}{8} \\
\frac{0}{\Sigma}\end{array}$ & 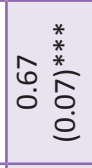 & 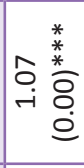 & $\begin{array}{l}\tilde{I} \\
-1 \\
\vdots \\
0 \\
\infty \\
\infty \\
0 \\
0\end{array}$ & م) & 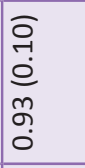 & ' & $\begin{array}{l}\frac{J}{\mathrm{I}} \\
\frac{\mathrm{d}}{\mathrm{D}} \\
\frac{0}{\Sigma}\end{array}$ & 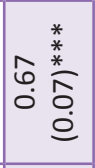 & 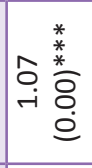 & $\begin{array}{l}\overline{-1} \\
-1 \\
0 \\
0 \\
0 \\
0\end{array}$ & 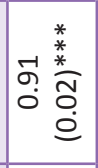 & 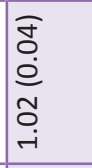 & \\
\hline 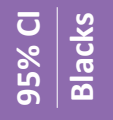 & & 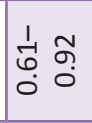 & مُ & & & 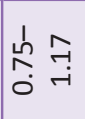 & ' & & $\begin{array}{lll}1 & -1 \\
0 & 0 \\
0 & \circ & 0\end{array}$ & $\begin{array}{ll}1 & \infty \\
0 & 0 \\
-i & \stackrel{-}{~}\end{array}$ & & & $\begin{array}{ll}1 & 0 \\
\infty & 0 \\
0 & - \\
0 & -\end{array}$ & ' \\
\hline $\begin{array}{l}\frac{\tilde{m}}{\underline{a}} \\
\frac{\alpha}{I}\end{array}$ & $\begin{array}{l}\frac{6}{2} \\
\frac{\pi}{0} \\
\frac{0}{\Sigma}\end{array}$ & 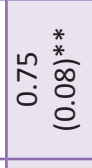 & \begin{aligned} & $\stackrel{*}{*} \\
\infty & \stackrel{*}{*} \\
$\hdashline & $\stackrel{*}{0} \\
& \stackrel{0}{0}\end{aligned}$ & & & $\begin{array}{l}0 \\
0 \\
-1 \\
0 \\
\infty \\
0 \\
0 \\
0\end{array}$ & ' & 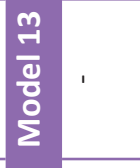 & 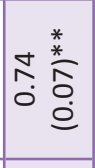 & 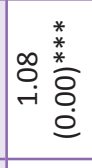 & & & $\begin{array}{l}\bar{y} \\
\dot{0} \\
\dot{0} \\
\bar{o} \\
0 \\
0\end{array}$ & ' \\
\hline ถั้ ర & & \begin{tabular}{ll}
1 & 0 \\
\multirow{f}{*}{} & 0 \\
0 & 0 \\
0 & 0
\end{tabular} & $\begin{array}{ll}1 & 0 \\
0 & \stackrel{-}{-} \\
-i & -1\end{array}$ & $\begin{array}{ll}1 & 0 \\
0 & 0 \\
0 & 0 \\
0 & 0\end{array}$ & 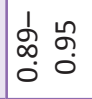 & 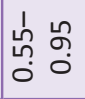 & ' & & \begin{tabular}{ll}
1 & $\tilde{1}$ \\
\multirow{f}{*}{} & 0 \\
0 & 0 \\
0
\end{tabular} & $\begin{array}{ll}1 & 0 \\
0 & \stackrel{-}{-} \\
-i & -\end{array}$ & $\begin{array}{ll}1 & n \\
& n \\
0 & 0 \\
0 & 0\end{array}$ & $\begin{array}{ll}1 & 0 \\
\infty & .0 \\
0 & 0 \\
0 & 0\end{array}$ & $\begin{array}{ll}1 & 0 \\
\infty & 0 \\
0 & 0 \\
0 & 0\end{array}$ & ' \\
\hline $\begin{array}{l}\tilde{\tilde{n}} \\
\stackrel{\alpha}{\underline{x}}\end{array}$ & $\begin{array}{l}\frac{n}{20} \\
\frac{0}{0} \\
\sum\end{array}$ & 苔 & 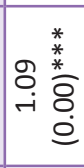 & 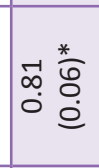 & م) & $\begin{array}{l}\hat{2} \\
\hat{0} \\
0 \\
0 \\
0 \\
0\end{array}$ & ' & $\begin{array}{l}\frac{N}{2} \\
\frac{10}{\circ} \\
\frac{0}{\Sigma}\end{array}$ & 苔 & & $\begin{array}{ll} & * \\
0 & \\
0 & 0 \\
0 & 0 \\
0 & 0 \\
0\end{array}$ & 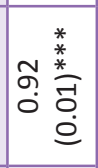 & $\begin{array}{ll}\infty & \stackrel{*}{*} \\
\infty & \stackrel{*}{\sigma} \\
0 & \stackrel{0}{0}\end{array}$ & \\
\hline $\begin{array}{l}\text { च } \\
\text { ถัँ }\end{array}$ & & $\begin{array}{ll}1 & \\
11 & 0 \\
& 0 \\
0 & 0\end{array}$ & 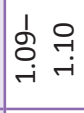 & & & \begin{tabular}{ll}
1 & 0 \\
\cline { 1 - 1 } & $\infty$ \\
0 & 0 \\
0 & 0
\end{tabular} & . & & 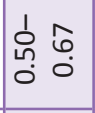 & 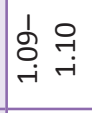 & & & \begin{tabular}{ll}
$b$ & \multicolumn{1}{c}{} \\
& \multicolumn{2}{c}{} \\
0 & 0
\end{tabular} & ' \\
\hline$\frac{\frac{\pi}{\tilde{n}}}{\frac{\alpha}{I}}$ & $\begin{array}{l}\frac{+}{80} \\
\frac{0}{\circ} \\
\frac{0}{\Sigma}\end{array}$ & 童 & 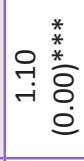 & & & 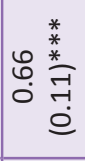 & ' & $\begin{array}{l}\frac{-1}{7} \\
\frac{1}{10} \\
\frac{0}{2} \\
\frac{0}{2}\end{array}$ & 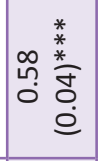 & 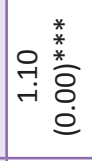 & & & 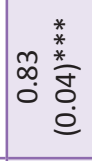 & \\
\hline ओั ర & $\begin{array}{ll}1 & 0 \\
0 & 0 \\
- & 0 \\
- & -\end{array}$ & $\begin{array}{ll}1 & 0 \\
\text { gे } \\
\text { o. }\end{array}$ & {$\left[\begin{array}{ll}1 & 0 \\
0 & 8 \\
-i & \stackrel{-}{-}\end{array}\right.$} & 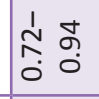 & $\begin{array}{ll}1 & + \\
0 & 0 \\
0 & 0 \\
0 & 0\end{array}$ & $\begin{array}{l}1 \\
\hat{n} \\
\text { นุ } \\
0 \\
0\end{array}$ & $\underset{i}{1} \stackrel{\infty}{\stackrel{\infty}{-}}$ & 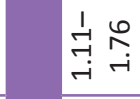 & $\begin{array}{ll}1 & 0 \\
\text { g. } \\
\text { o. } \\
0\end{array}$ & 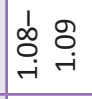 & $\begin{array}{ll}1 & n \\
m & n \\
0 & 0 \\
0 & 0\end{array}$ & \begin{tabular}{ll}
1 & \multicolumn{1}{c}{} \\
$\infty$ &. \\
0 & 0 \\
0
\end{tabular} & 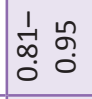 & 岂 \\
\hline 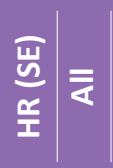 & 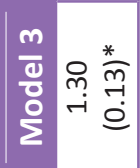 & 昼 & 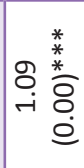 & 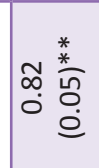 & م & 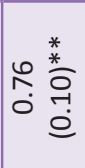 & $\begin{array}{cc}0 & * \\
m & \stackrel{*}{0} \\
-i & \stackrel{1}{0} \\
-i & 0\end{array}$ & 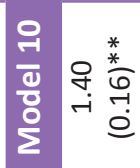 & 足 & & $\begin{array}{c}m \\
\infty \\
0 \\
0 \\
0\end{array}$ & 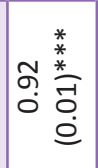 & 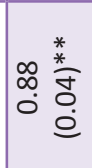 & 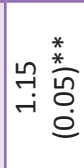 \\
\hline ํํ ڤั บ & 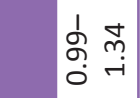 & $\begin{array}{ll}1 & 0 \\
\text { gे } \\
0 \\
0 & 0\end{array}$ & $\begin{array}{ll}1 & 0 \\
0 & 8 \\
-i & 0\end{array}$ & 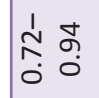 & 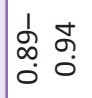 & $\begin{array}{ll}1 & 0 \\
0 & 0 \\
0 & 8 \\
0 & -\end{array}$ & . & \begin{tabular}{ll}
1 & $m$ \\
\hdashline & $m$ \\
0 & $m$
\end{tabular} & $\begin{array}{ll}1 & n \\
g & 0 \\
0 & 0 \\
0 & 0\end{array}$ & $\begin{array}{ll}1 & 0 \\
0 & \stackrel{8}{-} \\
-i & \stackrel{-}{-}\end{array}$ & $\begin{array}{ll}1 & n \\
m & n \\
0 & 0 \\
0 & 0\end{array}$ & 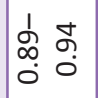 & $\begin{array}{ll}1 & \infty \\
\infty & \infty \\
0 & 0 \\
0 & 0\end{array}$ & ' \\
\hline 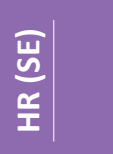 & 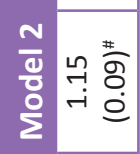 & 草 & $\begin{array}{rl} & \stackrel{*}{*} \\
0 & \stackrel{*}{*} \\
-i & 0 \\
0\end{array}$ & $\underset{\substack{\infty \\
0 \\
0}}{\stackrel{*}{*}}$ & م) & 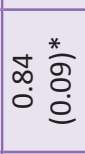 & ' & 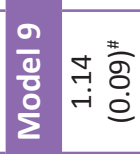 & 织 & 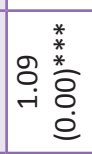 & 䓪 & م) & 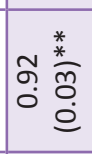 & ' \\
\hline ڤั่ ত & 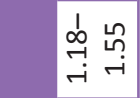 & $\begin{array}{ll}1 & \infty \\
& 0 \\
0.0 & 0 \\
0 & 0\end{array}$ & 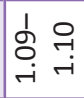 & & & 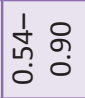 & , & 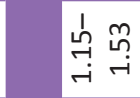 & 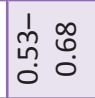 & 南 & & & $\begin{array}{cc}1 & n \\
0 & 0 \\
0 & 0 \\
0 & 0\end{array}$ & ' \\
\hline 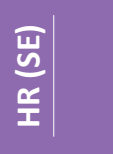 & 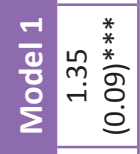 & 草 & $\begin{array}{r}\stackrel{*}{*} \\
0 \stackrel{*}{*} \\
-i \\
\stackrel{*}{0}\end{array}$ & & & $\begin{array}{ll}m & * \\
& \stackrel{*}{*} \\
0 & 0 \\
0 & 0 \\
& 0\end{array}$ & ' & 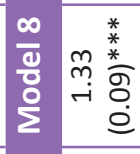 & o & $\begin{array}{l}\stackrel{*}{*} \\
\stackrel{*}{*} \\
\stackrel{*}{8} \\
\stackrel{0}{0}\end{array}$ & & & 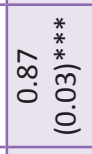 & \\
\hline 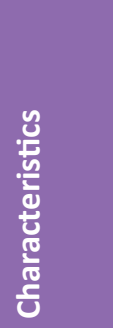 & 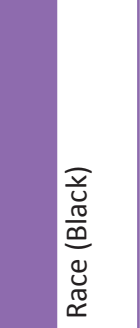 & 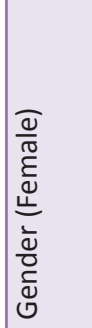 & 总 & 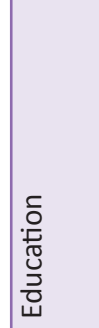 & $\begin{array}{l}\stackrel{0}{E} \\
\text { है } \\
\underline{\underline{G}}\end{array}$ & 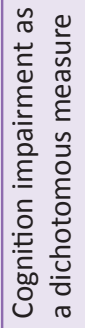 & 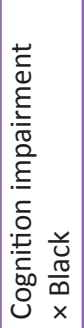 & 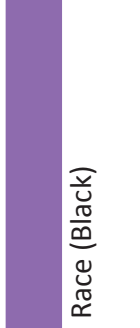 & 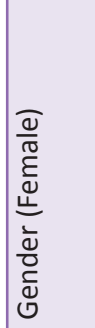 & $\underset{\mathbb{<}}{\stackrel{8}{0}}$ & 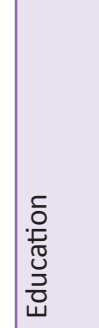 & 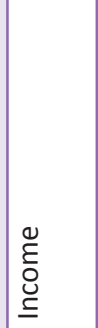 & 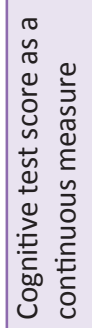 & 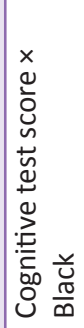 \\
\hline
\end{tabular}




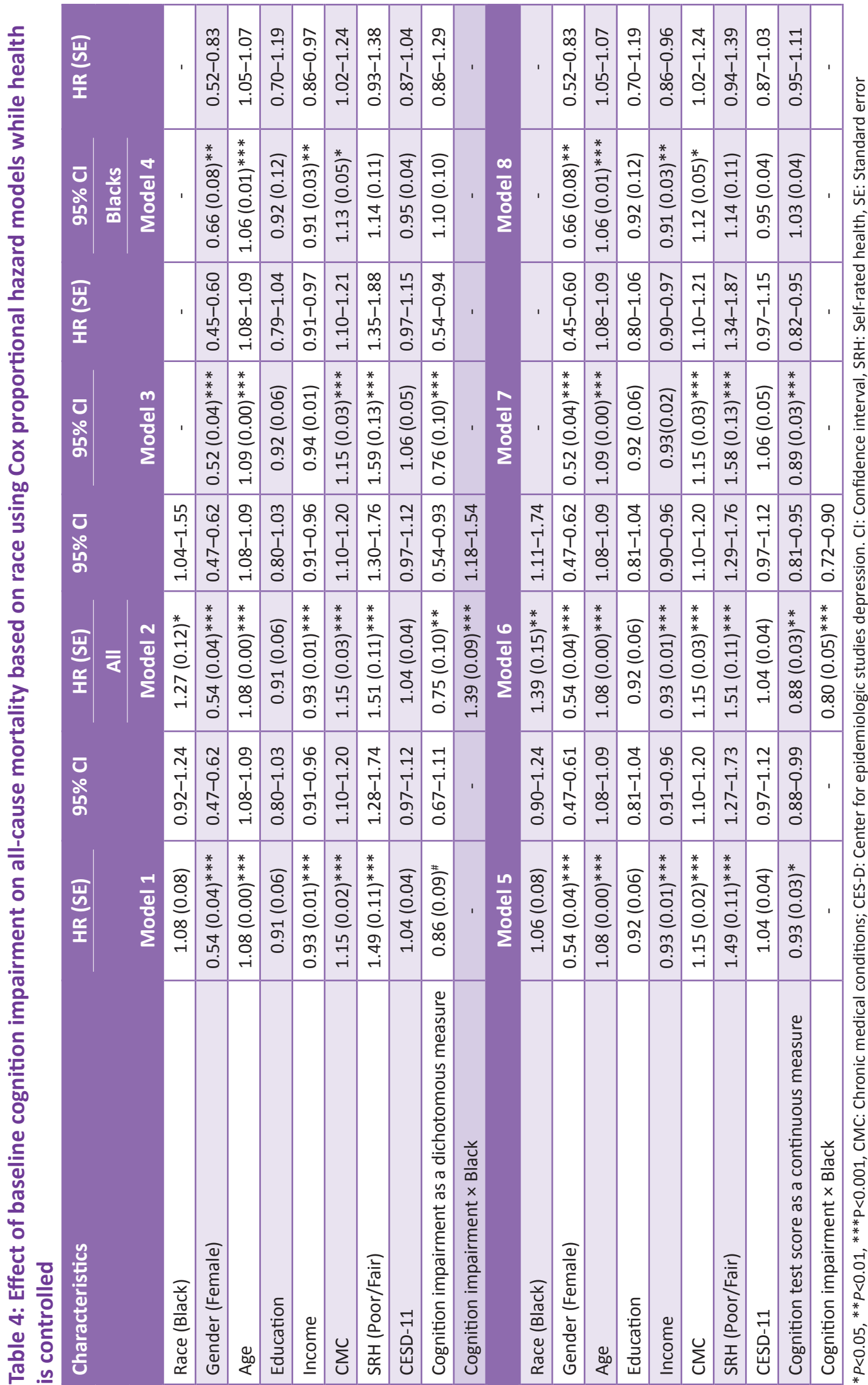


as stated by Moody-Ayers et al. in 2005, "efforts to understand cognitive function may enhance our understanding of Black-White disparities in health outcomes ${ }^{[11]}$."

The Black-White variation in the predictive role of baseline cognitive score on mortality may be considered in line with some other studies suggesting that baseline characteristics fail to predict long-term mortality among Blacks. ${ }^{[12,13,31]}$ In two studies in 2015, baseline SRH and depression, which predict mortality of Whites failed to predict the same outcome for Blacks. ${ }^{[12,13]}$ In 2001 Ferraro and Kelley-Moore showed that although SRH as a time-varying covariate predicts mortality among Blacks, baseline SRH is not as good predictor of long-term mortality for Blacks as Whites. ${ }^{[31]}$ Other studies have also shown that SRH may lose its predictive validity for mortality among individuals with lower socio-economic status. ${ }^{[32,33]}$ This is again in support of our findings, given race is a strong proxy of social class and socio-economic status in US. ${ }^{[34]}$

Regarding the main effect of race on cognitive ability, a number of studies in U.S. have shown that Blacks have lower unadjusted scores on cognitive function than Whites. ${ }^{[1,35]}$ This difference is largely due to lower socio-economic status ${ }^{[35]}$ and also an accelerated decline in health ${ }^{[36]}$ among Blacks compared to Whites. In both races, higher socioeconomic status indicators such as education was associated with lower cognitive decline, a finding which is supported by the literature. ${ }^{[37,38]}$ It has been shown that years of schooling is a strong predictor of cognitive reserve. ${ }^{[39]}$

Research on the association between baseline cognitive impairment, cognitive decline over time, and long-term mortality has provided mixed findings. ${ }^{[6]}$ While the effect of severe cognitive impairment on mortality stays significant above and beyond the effects of demographic, socio-economic, and health status, ${ }^{[40-43]}$ the long-term effect of mild cognitive impairment at baseline as a predictor of mortality may be more debatable, as shown only by some ${ }^{[44,45]}$ but not all ${ }^{[40,46]}$ studies. Similarly, findings are mixed regarding the effect of cognitive decline over time on subsequent mortality, which is shown by some ${ }^{[40]}$ but not all ${ }^{[41]}$ studies.

Among Whites, a higher risk of mortality with cognitive impairment at baseline was independent of demographics, socio-economic status, physical health, and depressive symptoms. This finding is in line with the literature. ${ }^{[3,5,6]}$ The previous population-based studies have shown that the risk of death is about $50 \%$ higher among those with low cognitive test scores at baseline. ${ }^{[47-50]}$

Among Blacks, however, baseline cognitive test score failed to predict long-term all-cause mortality similarly in the absence or presence of baseline demographics, socio-economics, and health status. Interestingly, some, ${ }^{[51,52]}$ if not all[ ${ }^{[7,53]}$ studies, have shown higher survival rates of Blacks with Alzheimer's disease compared to their White counterparts. In a study among individuals with Alzheimer's disease, cognitive impairment had a stronger effect on mortality among Blacks than Whites, which is in contrast to our results. ${ }^{[53]}$

We showed that Black-White differences in the cognitive function - mortality link are not due to racial differences in baseline socio-economic or health status, as the interaction between race and baseline cognitive test score on mortality remained significant with potential confounders (or mediators) in the model. The exact mechanism by which baseline cognitive ability predicts mortality is still unknown. As poor health status is associated with both cognitive impairment and mortality, it may confound or mediate the cognitive ability - mortality association. Several chronic medical conditions that adversely affect cognitive test scores ${ }^{[54]}$ also increases the risk of mortality. ${ }^{[33,55]}$ Longitudinal studies have shown that cognitive impairment at baseline predicts incident dementia, ${ }^{[56]}$ which reduces survival. ${ }^{[9,55]}$ According to the population-based studies, ${ }^{[57-74]}$ individuals with Alzheimer's disease are up to 3 times more likely to die than those without the condition. ${ }^{[53]}$ Thus, studies on the link between cognition and mortality require adjustment for chronic diseases. As explained above, higher socio-economic status is another major determinant of cognitive function inside and across populations. ${ }^{\text {[37-39] }}$

The result of our study has clinical and public health implications. Cognitive impairment is a modifiable risk factor for premature mortality, and some policies and interventions such as education may improve cognitive functioning of the most atrisk populations. ${ }^{[51,53,75-77]}$ For instance, the ACTIVE trial has shown significant benefits for memory, reasoning, and processing speed training at 5 and even 10 year follow-ups in cognitively intact older adults. ${ }^{[76,77]}$ However, some methodological issues of the ACTIVE study should be addressed. [78] Despite this, some promising results have suggested that cognitive function can be enhanced. 
[79-84] As cognitive ability plays a different role in predicting mortality of Blacks and Whites, ${ }^{[5,7,8]}$ interventions that enhance cognitive reserve of the communities may differently benefit populations' survival. Design and development of communitybased and clinical interventions that use cognitive maintenance/enhancement as a strategy to reduce mortality of populations will be improved by similar research-based information on the magnitude of the relationship between cognitive test score and long-term mortality across population groups. Such information may be used to decide between universal or population-specific programs that wish to promote longevity of the public through enhancement of cognitive reserve. ${ }^{[55]}$

Our findings are relevant to the complex causes the Black-White economic and health gap in the United States. ${ }^{[11]}$ We argue that it is not race or risk factors but race and risk factors that cause disparities. In a study in 2005, Moody-Ayers et al. measured cognitive function and functional decline among 779 Black and 4892 White community samples of older adults (age 70 or older) and showed that cognitive function might mediate the higher functional decline among Blacks. ${ }^{[55]}$

The findings are also in line with the Black-White health paradox, defined as a disproportionately lower prevalence of a wide range of undesired subjective health outcomes, despite higher levels of objective health problems such as chronic disease among Black Americans, in comparison to White Americans. ${ }^{[17,64-68]}$ Based on the Black-White health paradox, Blacks do not show some of the expected associations that are commonly found among Whites. ${ }^{[17,64-68]}$ The results are also in line with the Minorities Diminished Returns theory ${ }^{\text {[85-93] }}$ and also other studies that have shown resources better serve Whites than Blacks, ${ }^{[12-14]}$ and that the predictive role of baseline risk and protective factors on long-term outcomes are larger for Whites than Blacks. These patterns may be due to biases in measurement, ${ }^{[94]}$ or racism. ${ }^{\text {[95-99] }}$

Our study had some limitations that need attention. First, cognitive ability is subject to change; however, this study did not model cognitive decline over time. This is particularly important because both baseline and decline in cognitive ability are shown to predict mortality. ${ }^{[69-72]}$ Another limitation of this study is how we measured cognitive function, which may cause some measurement bias, which may itself depend on culture, ethnicity, or race. ${ }^{[35,73,74]}$ The study did not control for specific chronic neurological (e.g., dementia, and Alzheimer's disease) or psychiatric disorders (e.g., major clinical depression) conditions that impair cognitive abilities. Using a nationally representative sample and having a 25 year follow-up period were, however, major strengths of this study.

\section{Conclusions}

Baseline cognitive test score predicts long-term all-cause mortality among Whites but not Blacks. Future research should explore factors that may explain why minority ethnic differences exist in the role of cognitive test score on all-cause mortality risk in the United States. It is likely that socio-economics, physical health, and mental health factors differently confound or mediate the links between baseline cognitive test scores and subsequent mortality across race. Culture, access to health care, resource utilization, pattern of comorbid conditions, and health risk behaviors are also other potential explanatory factors behind Black-White differences in the cognitive test score mortality link.

\section{Author Contributions}

Shervin Assari designed and analyzed this work and drafted the manuscript and conducted the revisions. Shervin Assari had full access to all of the data in the study and takes responsibility for the integrity of the data and the accuracy of the data analysis.

\section{Acknowledgments}

In this section, you can acknowledge any support given which is not covered by the author contribution or funding sections. This may include administrative and technical support, or donations in kind (e.g., materials used for experiments).

\section{References}

1. Salthouse TA. What do adult age differences in the digit symbol substitution test reflect? J Gerontol 1992;47:121-8.

2. Royall DR, Lauterbach EC, Kaufer D, Malloy P, Coburn KL, Black KJ; Committee on Research of the American Neuropsychiatric Association. The cognitive correlates of functional status: A review from the committee on research of the American Neuropsychiatric Association. J Neuropsychiatry Clin Neurosci 2007;19:249-65.

3. Lavery LL, Dodge HH, Snitz B, Ganguli M. Cognitive decline and mortality in a community-based cohort: 
The Monongahela valley independent elders survey. J Am Geriatr Soc 2009;57:94-100.

4. Iwasa H, Gondo $\mathrm{Y}$, Yoshida $\mathrm{Y}$, Kwon J, Inagaki H, Kawaai $\mathrm{C}$, et al. Cognitive performance as a predictor of functional decline among the non-disabled elderly dwelling in a Japanese community: A 4-year population-based prospective cohort study. Arch Gerontol Geriatr 2008;47:139-49.

5. Johnson JK, Lui LY, Yaffe K. Executive function, more than global cognition, predicts functional decline and mortality in elderly women. J Gerontol A Biol Sci Med Sci 2007;62:1134-41.

6. Nguyen HT, Black SA, Ray LA, Espino DV, Markides KS. Cognitive impairment and mortality in older Mexican Americans. J Am Geriatr Soc 2003;51:178-83.

7. Rosano C, Newman AB, Katz R, Hirsch CH, Kuller LH. Association between lower digit symbol substitution test score and slower gait and greater risk of mortality and of developing incident disability in well-functioning older adults. J Am Geriatr Soc 2008;56:1618-25.

8. Swan GE, Carmelli D, LaRue A. Performance on the digit symbol substitution test and 5-year mortality in the Western Collaborative Group Study. Am J Epidemiol 1995;141:32-40.

9. Dewey ME, Saz P. Dementia, cognitive impairment and mortality in persons aged 65 and over living in the community: A systematic review of the literature. Int J Geriatr Psychiatry 2001;16:751-61.

10. Cano C, Samper-Ternent R, Al Snih S, Markides K, Ottenbacher KJ. Frailty and cognitive impairment as predictors of mortality in older Mexican Americans. J Nutr Health Aging 2012;16:142-7.

11. Moody-Ayers SY, Mehta KM, Lindquist K, Sands L, Covinsky KE. Black-white disparities in functional decline in older persons: The role of cognitive function. J Gerontol A Biol Sci Med Sci 2005;60:933-9.

12. Assari S, Lankarani MM, Burgard S. Black white difference in long term predictive power of selfrated health on all-cause mortality in United States. Ann Epidemiol 2015;26:106-14.

13. Assari S, Burgard S. Black-white differences in the effect of baseline depressive symptoms on deaths due to renal diseases: 25 year follow up of a nationally representative community sample. J Renal Injury Prev 2015;4:127-34.

14. Assari S. Hostility, anger, and cardiovascular mortality among blacks and whites. Res Cardiovasc Med 2015;4:127-34.

15. House JS, Lepkowski JM, Kinney AM, Mero RP, Kessler RC, Herzog AR. The social stratification of aging and health. J Health Soc Behav 1994;35:213-34.

16. House JS, Kessler RC, Herzog AR. Age, socioeconomic status, and health. Milbank Q 1990;68:383-411.

17. Assari S. Zivin K. Burgard S. Long-term reciprocal associations between depressive symptoms and number of chronic medical conditions: Longitudinal support for black? White health paradox. J Racial Ethnic Health Dispar 2015;2:589-97.

18. McDowell I. Measuring Health: A Guide to Rating Scales and Questionnaires. New York: Oxford University Press; 2006.

19. Pfeiffer E. A short portable mental status questionnaire for the assessment of organic brain deficit in elderly patients. J Am Geriatr Soc 1975;23:433-41.

20. Xu M, Thomas PA, Umberson D. Marital quality and cognitive limitations in late life. J Gerontol Ser B Psychol Sci Soc Sci 2015;71:165-76.

21. Gallo JJ. Handbook of Geriatric Assessment. Burlington, Massachusetts: Jones and Bartlett Learning; 2006.

22. Chang $\mathrm{YW}$, Chen $\mathrm{WL}$, Lin FG, Fang $\mathrm{WH}$, Yen $\mathrm{MY}$, Hsieh CC, et al. Frailty and its impact on healthrelated quality of life: $A$ cross-sectional study on elder community-dwelling preventive health service users. PLoS One 2012;7:e38079.

23. DeSalvo KB, Bloser N, Reynolds K, He J, Muntner P. Mortality prediction with a single general self-rated health question. A meta-analysis. J Gen Intern Med 2006;21:267-75.

24. Kondo N, Sembajwe G, Kawachi I, van Dam RM, Subramanian SV, Yamagata Z. Income inequality, mortality, and self rated health: Meta-analysis of multilevel studies. BMJ 2009;339:b4471.

25. Idler EL, Benyamini Y. Self-rated health and mortality: A review of twenty-seven community studies. J Health Soc Behav 1997;38:21-37.

26. Lundberg $\mathrm{O}$, Manderbacka K. Assessing reliability of a measure of self-rated health. Scand J Soc Med 1996;24:218-24.

27. Radloff LS. The CES-D scale: A self-report depression scale for research in the general population. Appl Psychol Meas 1977;1:385-401.

28. Amtmann D, Kim J, Chung H, Bamer AM, Askew RL, Wu S, et al. Comparing CESD-10, PHQ-9, and PROMIS depression instruments in individuals with multiple sclerosis. Rehabil Psychol 2014;59:220-9.

29. Zhang $\mathrm{W}$, O'Brien N, Forrest Jl, Salters KA, Patterson TL, Montaner JS, et al. Validating a shortened depression scale (10 item CES-D) among HIV-positive people in British Columbia, Canada. PLoS One 2012;7:e40793.

30. Andresen EM, Malmgren JA, Carter WB, Patrick DL. Screening for depression in well older adults: Evaluation of a short form of the CES-D (center for epidemiologic studies depression scale). Am J Prev Med 1994;10:77-84.

31. Ferraro KF, Kelley-Moore JA. Self-rated health and mortality among black and white adults: Examining the dynamic evaluation thesis. J Gerontol B Psychol Sci Soc Sci 2001;56:S195-205.

32. Dowd JB, Zajacova A. Does the predictive power of self-rated health for subsequent mortality risk vary 
by socioeconomic status in the US? Int J Epidemiol 2007;36:1214-21.

33. Regidor E, Guallar-Castillón P, Gutiérrez-Fisac JL, Banegas JR, Rodríguez-Artalejo F. Socioeconomic variation in the magnitude of the association between self-rated health and mortality. Ann Epidemiol 2010;20:395-400.

34. Williams DR, Sternthal M. Understanding racial-ethnic disparities in health: Sociological contributions. J Health Soc Behav 2010;51 Suppl:S15-27.

35. Carle AC, Weech-Maldonado R, Ngo-Metzger Q, Hays RD. Evaluating measurement equivalence across race and ethnicity on the CAHPS cultural competence survey. Med Care 2012;50 9 Suppl 2:S32-6.

36. Sachs-Ericsson N, Blazer DG. Racial differences in cognitive decline in a sample of community-dwelling older adults: The mediating role of education and literacy. Am J Geriatr Psychiatry 2005;13:968-75.

37. Fei $M, Q u ~ Y C$, Wang T, Yin J, Bai JX, Ding QH. Prevalence and distribution of cognitive impairment no dementia (CIND) among the aged population and the analysis of socio-demographic characteristics: The community-based cross-sectional study. Alzheimer Dis Assoc Disord 2009;23:130-8.

38. Shih RA, Ghosh-Dastidar B, Margolis KL, Slaughter ME, Jewell A, Bird CE, et al. Neighborhood socioeconomic status and cognitive function in women. Am J Public Health 2011;101:1721-8.

39. Farfel JM, Nitrini $R$, Suemoto CK, Grinberg LT, Ferretti RE, Leite RE, et al. Very low levels of education and cognitive reserve: $A$ linicopathologic study. Neurology 2013;81:650-7.

40. Bassuk SS, Wypij D, Berkman LF. Cognitive impairment and mortality in the communitydwelling elderly. Am J Epidemiol 2000;151:676-88.

41. Bruce ML, Hoff RA, Jacobs SC, Leaf PJ. The effects of cognitive impairment on 9-year mortality in a community sample. J Gerontol B Psychol Sci Soc Sci 1995;50B:289-96.

42. Jorm AF, Henderson AS, Kay DK, Jacomb PA. Mortality in relation to dementia, depression, and social integration in an elderly community sample. Int J Geriatr Psychiatry 1991;6:5-11.

43. Shapiro E, Tate RB. The impact of a mental status score and a dementia diagnosis on mortality and institutionalization. J Aging Health 1991;3:28-46.

44. Kelman HR, Thomas C, Kennedy GJ, Cheng J. Cognitive impairment and mortality in older community residents. Am J Public Health 1994;84:1255-60.

45. Marquis S, Moore MM, Howieson DB, Sexton G, Payami $\mathrm{H}$, Kaye JA, et al. Independent predictors of cognitive decline in healthy elderly persons. Archives of neurology. 2002;59:601-6.

46. Jagger C, Clarke M. Mortality risks in the elderly: Five-year follow-up of a total population. Int J
Epidemiol 1988;17:111-4.

47. Johansson B, Zarit SH. Early cognitive markers of the incidence of dementia and mortality: A longitudinal population-based study of the oldest old. Int J Geriatr Psychiatry 1997;12:53-9.

48. Palmer K, Wang HX, Backman L, Winblad B, Fratiglioni L. Differential evolution of cognitive impairment in nondemented older persons: Results from the Kungsholmen project. Am J Psychiatry 2002;159:436-42.

49. Ingles JL, Fisk JD, Merry HR, Rockwood K. Five-year outcomes for dementia defined solely by neuropsychological test performance. Neuroepidemiology 2003;22:172-8.

50. Guehne U, Luck T, Busse A, Angermeyer M, RiedelHeller SG. Mortality in individuals with mild cognitive impairment: Results of the Leipzig longitudinal study of the aged (LEILA75). Neuroepidemiology 2007;29:226-34.

51. Edwards JD, Wadley VG, Vance DE, Wood K, Roenker DL, Ball KK. The impact of speed of processing training on cognitive and everyday performance. Aging Ment Health 2005;9:262-71.

52. Wadley VG, Benz RL, Ball KK, Roenker DL, Edwards JD, Vance DE. Development and evaluation of homebased speed of- processing training for older adults. Arch Phys Med Rehabil 2006;87:757-63.

53. Wilson RS, Aggarwal NT, Barnes LL, Bienias JL, de Leon CF, Evans DA. Biracial population study of mortality in mild cognitive impairment and Alzheimer disease. Arch Neurol 2009;66:767-72.

54. Tombaugh TN, Mclntyre NJ. The mini-mental state examination: A comprehensive review. J Am Geriatr Soc 1992;40:922-35.

55. Iwasa H, Kai I, Yoshida Y, Suzuki T, Kim H, Yoshida H. Information processing speed and 8-year mortality among community-dwelling elderly Japanese. J Epidemiol 2014;24:52-9.

56. Rapp MA, Reischies FM. Attention and executive control predict Alzheimer disease in late life: Results from the Berlin aging study (BASE). Am J Geriatr Psychiatry 2005;13:134-41.

57. Evans DA, Smith LA, Scherr PA, Albert MS, Funkenstein $\mathrm{HH}$, Hebert LE. Risk of death from Alzheimer's disease in a community population of older persons. Am J Epidemiol 1991;134:403-12.

58. Katzman R, Hill LR, Yu ES, Wang ZY, Booth $A$, Salmon DP, et al. The malignancy of dementia: Predictors of mortality in clinically diagnosed dementia in a population survey of Shanghai, China. Arch Neurol 1994;51:1220-5.

59. Agüero-Torres H, Fratiglioni L, Guo Z, Viitanen M, Winbald B. Mortality from dementia in advanced age: A 5-year follow-up study of incident dementia cases. J Clin Epidemiol 1999;52:737-43.

60. Helmer C, Joly P, Letenneur L, Commenges D, Dartigues JF. Mortality with dementia: Results from

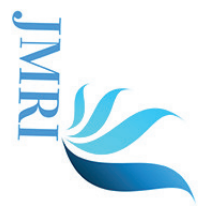


a French prospective community-based cohort. Am J Epidemiol 2001;154:642-8.

61. Tschanz JT, Corcoran C, Skoog I, Khachaturian AS, Herrick J, Hayden KM, et al. Dementia: The leading predictor of death in a defined elderly population: The Cache County Study. Neurology 2004;62:1156-62.

62. Fitzpatrick AL, Kuller LH, Lopez OL, Kawas $\mathrm{CH}$, Jagust W. Survival following dementia onset: Alzheimer's disease and vascular dementia. J Neurol Sci 2005;229-230:43-9.

63. Ganguli M, Dodge HH, Shen C, Pandav RS, DeKosky ST. Alzheimer's disease and mortality: A 15-year epidemiological study. Arch Neurol 2005;62:779-84.

64. Kim G, DeCoster J, Chiriboga DA, Jang Y, Allen RS, Parmelee P. Associations between self-rated mental health and psychiatric disorders among older adults: Do racial/ethnic differences exist? Am J Geriatr Psychiatry 2011;19:416-22.

65. Jang Y, Park NS, Kang SY, Chiriboga DA. Racial/ethnic differences in the association between symptoms of depression and self-rated mental health among older adults. Community Ment Health J 2014;50:325-30.

66. Korhonen $P E$, Kautiainen $H$, Mäntyselkä P. Screening for cardiovascular risk factors and self-rated health in a community setting: A cross-sectional study in Finland. Br J Gen Pract 2014;64:e611-5.

67. Keyes, Corey LM. The black white paradox in health: Flourishing in the face of social inequality and discrimination. J Pers 2009;77:1677-706.

68. Barnes DM, Keyes KM, Bates LM. Racial differences in depression in the United States: How do subgroup analyses inform a paradox. Soc Psychiatry Psychiatr Epidemiol 2013;48:1941-9.

69. Shipley BA, Der G, Taylor MD, Deary IJ. Cognition and all-cause mortality across the entire adult age range: Health and lifestyle survey. Psychosom Med 2006;68:17-24.

70. Shipley BA, Der G, Taylor MD, Deary IJ. Cognition and mortality from the major causes of death: The Health and Lifestyle Survey. J Psychosom Res 2008;65:143-52.

71. Shipley BA, Der G, Taylor MD, Deary IJ. Association between mortality and cognitive change over 7 years in a large representative sample of UK residents. Psychosom Med 2007;69:640-50.

72. Richards M1, Shipley B, Fuhrer R, Wadsworth ME. Cognitive ability in childhood and cognitive decline in mid-life: Longitudinal birth cohort study. BMJ 2004;328:552.

73. Blankson AN, McArdle JJ. A brief report on the factor structure of the cognitive measures in the HRS/ AHEAD studies. J Aging Res 2014;2014:798514.

74. Slater $\mathrm{CH}$, Lorimor RJ, Lairson DR. The independent contributions of socioeconomic status and health practices to health status. Prev Med 1985;14:372-8.

75. Feinstein JS. The relationship between socioeconomic status and health: A review of the literature. Milbank Q 1993;71:279-322.

76. Rebok GW, Ball K, Guey LT, Jones RN, Kim HY, King JW, et al. Ten-year effects of the advanced cognitive training for independent and vital elderly cognitive training trial on cognition and everyday functioning in older adults. J Am Geriatr Soc 2014;62:16-24.

77. Ball K, Berch DB, Helmers KF, Jobe JB, Leveck MD, Marsiske $M$, et al. Effects of cognitive training interventions with older adults: A randomized controlled trial. JAMA 2002;288:2271-81.

78. Willis SL, Tennstedt SL, Marsiske M, Ball K, Elias J, Koepke KM, et al. Long-term effects of cognitive training on everyday functional outcomes in older adults. JAMA 2006;296:2805-14.

79. Hampstead BM, Gillis MM, Stringer AY. Cognitive rehabilitation of memory for mild cognitive impairment: A methodological review and model for future research. J Int Neuropsychol Soc 2014;20:135-51.

80. Hampstead BM, Sathian K, Moore AB, Nalisnick C, Stringer AY. Explicit memory training leads to improved memory for face-name pairs in patients with mild cognitive impairment: Results of a pilot investigation. J Int Neuropsychol Soc 2008;14:883-9.

81. Hampstead BM, Stringer AY, Stilla RF, Giddens M, Sathian K. Mnemonic strategy training partially restores hippocampal activity in patients with mild cognitive impairment. Hippocampus 2012;22:1652-8.

82. Hampstead BM, Sathian K, Phillips PA, Amaraneni A, Delaune WR, Stringer AY. Mnemonic strategy training improves memory for object location associations in both healthy elderly and patients with amnestic mild cognitive impairment: A randomized, singleblind study. Neuropsychology 2012;26:385-99.

83. Hampstead BM, Stringer AY, Stilla RF, Deshpande G, $\mathrm{Hu} X$, Moore $A B$, et al. Activation and effective connectivity changes following explicit-memory training for face-name pairs in patients with mild cognitive impairment: A pilot study. Neurorehabil Neural Repair 2011;25:210-22.

84. Hampstead BM, Sathian K, Bikson M, Stringer AY. Combined mnemonic strategy training and highdefinition transcranial direct current stimulation for memory deficits in mild cognitive impairment. Alzheimers Dement 2017;3:459-70.

85. Hampstead BM, Mosti CB, Swirsky-Sacchetti T. Cognitively-based methods of enhancing and maintaining functioning in those at risk of Alzheimer's disease. J Alzheimers Dis 2014;42 Suppl 4:S483-93.

86. Assari S. Unequal gain of equal resources across racial groups. Int J Health Policy Manag 2018;7:1-9.

87. Assari S. Health disparities due to blacks' diminished return: Public policy solutions. Soc Issues Policy Rev 2018;12:112-45.

88. Assari S, Lankarani MM. Race and urbanity alter the protective effect of education but not income on 


\section{Assari: Race, cognition, and mortality}

mortality. Front Public Health 2016;4:100.

89. Assari S, Thomas A, Caldwell CH, Mincy RB. Blacks' diminished health return of family structure and socioeconomic status; 15 years of follow-up of a national urban sample of youth. J Urban Health 2018;95:21-35.

90. Assari S. The benefits of higher income in protecting against chronic medical conditions are smaller for African Americans than whites. Healthcare 2018;6:2.

91. Assari S. Life expectancy gain due to employment status depends on race, gender, education, and their intersections. J Racial Ethn Health Disparities 2017; 5:375-86.

92. Assari S. General self-efficacy and mortality in the USA; racial differences. J Racial Ethn Health Disparities 2017;4:746-57.

93. Assari S. Whites but not blacks gain life expectancy from social contacts. Behav Sci (Basel) 2017;7:E68.

94. Assari S. Race, sense of control over life, and shortterm risk of mortality among older adults in the
United States. Arch Med Sci 2017;13:1233-40.

95. Rushton JP, Jensen AR. Thirty years of research on race differences in cognitive ability. Psychol Public Policy Law 2005;11:235.

96. Assari $\mathrm{S}, \mathrm{Caldwell} \mathrm{CH}$. Teacher discrimination reduces school performance of African American youth: Role of gender. Brain Sci 2018;8:183.

97. Assari S. Parental education attainment and educational upward mobility; role of race and gender. Behav Sci 2018;8:107.

98. Assari S. Race, intergenerational social mobility and stressful life events. Behav Sci 2018;8:86.

99. Assari S. Does school racial composition explain why high income black youth perceive more discrimination? A gender analysis. Brain Sci 2018;8:140.

100. Assari S, Lankarani MM. Workplace racial composition explains high perceived discrimination of high socioeconomic status African American men. Brain Sci 2018;8:139. 